\title{
ZUR TEXTKRITIK DER SPIELE VON YORK.
}

\section{Zu $1 / 3$.}

Im ersten der Yorker Spiele, welches die schöpfung und den fall der engel behandelt, beginnt der herr seine rede mit den worten:

I am gracyus and grete, god withoutyn begynnyng,

I am maker vnmade, all mighte es in me,

I am lyfe and way vnto welth wynnyng,

I am formaste and fyrste, als I byd sall it be.

Zum dritten vers bemerkt Holthausen Angl. XXI 443: 'Der rhythmus erfordert die einsetzung von pe vor way und von pat is nach welth'. Er will also lesen:

I am lyfe and [pe] way vnto welth [pat is] wynnyng,

d. h. er hält es für notwendig, streng anapästischen rhythmus herzustellen. Seine besserung trifft besonders den zweiten halbvers, wo er an dem fehlen der senkung zwischen den hebungen anstoss genommen hat. Aber wenn auch die meisten verse dieses spiels in dem tonfall verlaufen, den Holthausen herstellen will, so ist er durchaus nicht alleinherrschend. Speziell fehlen der senkung findet sich auch sonst; so im zweiten halbvers:

Ay with stedefaste steuen lat vs stánde stíll $\mathbf{7 5}$

Lorde! to be fede with pe fode of thi fáyre fáce 76

A! lorde, louid be thi name pat vs pis lighte lénte 121

The nighte euen fro pe day, so pat thai méte néuer 154;

im ersten:

And in $p^{e}$ fýrste fáythely, my thoghts to full-fyll 19

Euen to myne áwne fýgure pis blys to fulfyl 140

Aude in my fýrste mákyng to mustyr my mighte 145 . 
Dass etwa das ursprüngliche end-e von stande, fayre usw. noch gesprochen wurde, um eine senkungssilbe zu gewinnen, ist nicht glaublich, da sich sonst in diesem text nirgends eine spur von metrischer verwendung des $-e$ zeigt. Auch Holthausen muss dieser ansicht sein, denn in einem analogen fall aus einem anderen spiel (428/52, Angl. XXI 451, vgl. unten s. 389) sucht er die senkung nicht durch geltendmachung des - $e$ von harte ('herz'), sondern durch einschub einer vollsilbe $\mathrm{zu}$ gewinnen. Uebrigens wäre auch dem in rede stehenden vers auf diesem weg zu helfen gewesen, da welth, eine frühmittelengl. neubildung nach dem muster der abstracta auf - te aus ae. - đu wie strengthe aus ae. strengđu, ursprünglich - $-\ddot{e}$ hatte. Somit gehören alle angeführten fälle zusammen und wer einen aus rhythmischen gründen bessert, hätte meines erachtens auch die andern zu beseitigen.

Bleiben wir aber nicht bei empirischer betrachtungsweise stehen! Was für verse haben wir denn vor uns und nach welchen regeln sind sie gebaut? Es ist richtig: die vierhebigen zeilen, aus denen die ersten strophenhälften in diesem spiel bestehen, haben vorwiegend anapästischen rhythmus. Verse, denen dieser als das normale zu grunde liegt in dem sinn wie die gleichtaktigen metren prinzipiell jambischen rhythmus haben (Schipper, Grdr. II 1021), gab es nun aber im Mittelenglischen gar nicht. Anapästischer rhythmus stellte sich vielmehr immer nur sekundär ein, entweder vereinzelt indem in gleichtaktigen versen die einsilbigkeit der senkung nicht eingehalten wurde, oder - und das ist häufiger - im stabreimvers durch das vorwalten gewisser rhythmischer 'typen'. Verse dieser letzteren art haben wir nun klärlich vor uns, obwohl sie auch endreim zeigen, und wenn wir ihre rhythmische gestaltung beurteilen wollen, müssen wir von den regeln über den bau des mittelenglischen stabreimverses ausgehen.

In seiner reinsten gestalt tritt uns dieser ohne endreim entgegen, und da zeigt er eine deutliche weiterführung, wenn auch vereinfachung der eigentümlichkeiten des altenglischen stabreimverses (vgl. Grdr. Il 1009). Die zweite halbzeile verläuft wesentlich in drei typischen formen, die an gewissen stellen variierbar sind: $\mathrm{A}:(x){ }_{-}^{\prime} \times x(x){ }_{-}^{\prime}, \mathrm{C}: x \times(x)^{\prime}{ }_{-}^{\prime} \times$, und $\mathrm{BC}: x \times(x)^{\prime}-x^{\prime}-x$, sowie in varianten derselben, die 
durch verstummen der letzten senkung entstanden sind: $A^{1}$ : $(x)^{\prime}-x \times(x)^{\prime}, C^{1}: x \times(x)^{\prime}-1$ und $\mathrm{BC}^{1}: x \times(x)^{\prime}-x^{\prime}$. Was über diese typen hinausgeht oder unter ihnen zurückbleibt, kommt bei guten dichtern ganz vereinzelt vor und wird sichtlich gemieden. Im ersten halbvers zeigen sich ausser diesen formen noch vollere, die sich im wesentlichen als typus A mit mehrsilbigem auftakt oder stärker entwickelter mittel- oder endsenkung, nicht selten mit rhythmischen nebentönen versehen, charakterisieren lassen. Dass diese formulierungen von dem in manchen texten schwankenden wert des end-e nicht beeinträchtigt werden, habe ich erst kürzlich an anderer stelle neuerlich dargethan (Beibl. X $79 \mathrm{ff}$.). Da nun der typus A bei weitem überwiegt, so hat schon der reimfreie stabreimvers häufig einen wesentlich anapästischen rhythmus.

In noch höherem grade ist dies bei dem mit dem endreim versehenen der fall. Unter dem einfluss dieses den gleichtaktigen metren entnommenen bindemittels, welches offenbar auch die technischen schwierigkeiten der versbildung bedeutend steigerte, wurde der rhythmus gleichförmiger, indem der schon von haus aus häufigste typus noch mehr raum erhielt. Aber daneben sind die anderen nicht völlig unterdrückt, sondern treten immer wieder als gelegentliche varianten $\mathbf{z u}$ tage (Grdr. II 1014).

Wenn wir unter diesen gesichtspunkten die 80 vierhebigen verse unseres spiels, deren bau um so durchsichtiger ist, da das end-e sicher nicht mehr gilt, ins auge fassen, so finden ihre wechselnden formen ihre ungezwungene erklärung. Der typus A ( $\left.\mathrm{A}^{1}\right)$ überwiegt bei weitem, im ersten halbvers gewöhnlich mit zweisilbigem, im zweiten mit einsilbigem auftakt, so dass in der that nicht selten das schema

$$
x x^{\prime}-x x^{\prime}-x \| x^{\prime}-x x^{\prime}-(x)
$$

verwirklicht erscheint. Doch finden sich auch die anderen typen. Die belege für $\mathrm{C}_{\text {und }} \mathrm{C}^{1}(19 \mathrm{a}, 140 \mathrm{a}, 145 \mathrm{a} ; 3 \mathrm{~b}, 75 \mathrm{~b}$, $76 \mathrm{~b}, 121 \mathrm{~b}, 154 \mathrm{~b})$ sind die oben $\mathrm{s} .384$ bereits angezogenen fälle. Die für $\mathrm{BC}$, bez. $\mathrm{BC}^{1}$ sind im zweiten halbvers:

when pe féndes féll 148

und wohl auch

to rewárde on rówe 124 ;

im ersten halbvers: 
Here vndernéthe me nówe 25

Ay with stédefaste stéuen 75

ferner v. 28, 57, 89, 99, 105, 146.

Von wichtigkeit ist, dass aber auch die in der obigen formulierung gegebenen grenzen im ganzen noch gut eingehalten werden. Verse, die unter den normalmassen zurückbleiben, sind vereinzelt; es sind dies:

pe mighte of mé $18 \mathrm{~b}$

pat áre was lighte $100 \mathrm{~b}$

Thi ríghtwysnés 124 a.

Dasselbe gilt von solchen, die darüber hinausgehen. Zweite halbverse mit mehr als einsilbigem auftakt vor dem typus A, der im ersten halbvers typisch ist, kommen nur vereinzelt vor:

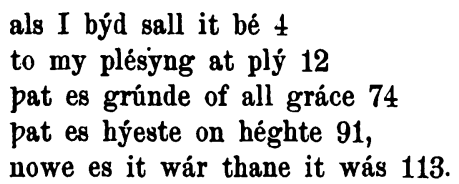

Von diesen fünf fällen schliessen sich übrigens drei $(4,74,91)$ an stumpf endigende erste halbzeilen an, so dass im zusammenhang des verses der zweisilbige auftakt immerhin weniger als solcher empfunden worden sein mag, und der letzte (113) gehört einer überhaupt schlecht überlieferten zeile an (vgl. Holthausen s. 444). Bedenkt man nun, dass im ersten halbvers fast die hälfte aller fälle (32) so gebaut sind, 'so erkennt man deutlich, dass die durch die 'typen' angegebenen grenzen dem metrischen gefühl des dichters lebendig waren.

Prüfen wir nun den von Holthausen emendierten vers, so finden wir, dass die überlieferte form zwar seltenere aber vollkommen berechtigte rhythmische varianten darstellt: im ersten halbvers den typus $\mathrm{BC}^{1}$, im zweiten $\mathrm{C}$, während in Holthausens herstellung der zweite halbvers vnto welth [bat is] wynnyng einen fall von A mit mehrsilbigem auftakt böte, eine form, die sichtlich gemieden wird. Somit ist vom rhythmischen standpunkt aus gegen die überlieferung nichts einzuwenden, während die emendation Holzhausens bedenklich erscheint.

Dazu kommt noch, dass inhaltlich meines erachtens die überlieferte lesart viel besser anspricht als die Holthausens. Welth heisst ja im Mittelenglischen nicht bloss reichtum, son- 
dern auch, seiner etymologie entsprechend, wohlbefinden, glück. So sagt im 24. Yorker Spiel Lazarus' schwester nach dessen tode: 'My welthe is went for euere' (198/155), und in den 'Abenteuern Arthurs' klagt der könig nach einer schweren verwundung: Alle the welthe of this worde thus a-way wytes (ed. Ritson, XVII 7). Das Promptorium Parvulorum glossiert geradezu: 'Welthe, or welfare Prosperitas, felicitas'. Im 16. jahrhundert ist diese bedeutung des wortes noch ganz lebendig (W. A. Wright, Bible Word Rook, Sec. Ed. 1884 s. v., A. Schmidt, Shakespeare-Lexikon s. v.), und in der kirchensprache hat sie sich bis auf den heutigen tag erhalten: die Litanei bietet die gegenüberstellung in all time of our tribulation, in all time of our wealth (Wright a. a. o.), wie auch in der Authorised Version der Bibel wealth and riches Ps. 112, 3 lat. gloria et divitiae wiedergiebt. Auch das compositum commonwealth ist hier anzuziehen.

Noch prägnanter wird aber die bedeutung des wortes in der christlichen terminologie: es bezeichnet geradezu seelenheil, ewige seligkeit. In der nordenglischen legendensammlung (Altengl. Leg. ed. Horstmann, N. F. 1881) schliesst der erzähler öfter mit dem wunsche, durch die hilfe des heiligen in die ewige seligkeit einzugehen, die dieser bereits geniesst, und da gebraucht er für diesen begriff wiederholt den ausdruck welth. So: He wis vs wiseli for to win Vnto pat welth pat he wons in $155 / 286$; ähnlich 148/440,173/768. Mein verehrter freund A. E. Schönbach belehrt mich überdies, dass der in frage stehende vers wohl an das herrenwort Joann. 14, 6 anknüpft: '(dicit ei Jesus): Ego sum via et veritas et vita; nemo venit ad Patrem, nisi por me'. Hier wurde via schlechtweg als via salutis gefasst; Act. Apost. 16, 17 heisst es von denen, die Christi lehre vortragen, einfach: qui annuntiant vobis viam salutis. Das nimmt die spätere kirchensprache auf: die christliche religion ist via salutis, Christus ist salvator. Auch in den psalmen ist Gott die quelle alles heiles (glückes) z. b. 26, 1: Dominus illuminatio mea et selus mea, quem timebo? Danach heisst der in rede stehende vers in der überlieferten lesart: 'ich bin das leben und der weg zur erlangung des heils', er giebt einfach die worte Christi Joann. 14, 6 nach der kirchlichen interpretation wieder. Warum der dichter nicht den gewöhnlichen ausdruck für heil, hele, gebraucht, ist nicht 
schwer zu ersehen: zu dem durch das bibelwort gegebene way benötigte er eine $w$-allitteration.

Auch gegen die hier vorliegende konstruktion ist nichts einzuwenden. Sie hat ihre seitenstücke in ausdrücken wie thorugh bedes biddynge, thorugh penyes delynge, wofür Mätzner III $^{3} 94$ belege bringt.

Wie Holthausen den sinn des verses fasst, ist mir nicht ganz klar. In seiner besserung muss wohl pat is wynnyng relativsatz im anschluss an welth sein: er scheint mir auf jeden fall ein seltsamer und matter zusatz, uud unmöglich ist er, wenn meine darlegungen über die bedeutung von welth an dieser stelle das richtige getroffen haben.

Ich glaube somit, dass wir keinen grund haben, von der überlieferung abzuweichen, sondern vielmehr allen anlass, an ihr festzuhalten. Man kann nur im zweifel sein, ob nicht aus syntaktischen gründen im ersten halbvers der artikel zu ergänzen wäre: I am [pe] lyfe and [be] way. Metrisch wäre diese besserung gleichgiltig.

\section{Zu 428/52; 363/132, 367/254, 369/325, 379/368.}

Aehnliche einwände sind auch gegen einige andere besserungen Holthausens zu erheben. Das 40. spiel, vom gang nach Emaus, ist im selben metrum geschrieben wie das erste. Wenn daher Holthausen in v. 52 (s. 428)

Whanne pat a spétyfful spére vn-to his hárte ránne

lesen will to his harte[-root] ranne (eb. s. 451), so übersieht er wieder eine eigentümlichkeit des stabreimverses. Wir haben vielmehr den typus $\mathrm{C}^{\prime}$ vor uns, wie er auch sonst in diesem spiel vorkommt (74 b, $106 \mathrm{~b}, 173 \mathrm{~b}, 28 \mathrm{a})$. Gänzliches fehlen der allitteration im zweiten halbvers (die übrigens auch trotz der emendation sehr mangelhaft wäre) ist ebenfalls nicht unerhört (vgl. v. 75, 182, 186, 193). Es ist somit kein grund vorhanden, das zusammenstossen der hebungen zu beseitigen. Ja, nicht einmal zur streichung des $v n$ - sind wir berechtigt. Die eingangssenkung der typen $\mathrm{C}$ und $\mathrm{BC}\left(\mathrm{C}^{1}\right.$ und $\mathrm{BC}^{\prime}$ ) kann gerade so wie die mittelsenkung von $\mathrm{A}\left(\mathrm{A}^{\prime}\right)$ auch mehr als zweisilbig sein (wie in diesem stück in v. $28 \mathrm{a}$ ). Unserem modernen rhythmischen gefühl ist ja ein vers der form $x \times \times{ }^{\prime}-1$ sehr auffällig und fremdartig. Aber wer sich 
in den mittelenglischen stabreimvers mit seinem eigentümlich unruhigen, aber um so eindringlicheren rhythmus einliest, wird bald von dieser empfindung frei werden und auch rein gefühlsmässig begreifen, was sich zunächst durch statistische untersuchung des wortgebrauches ergiebt.

Diese bemerkungen leiten uns zu einer gruppe von anderen fällen über, wo Holthausen durch streichung einer silbe rein anapästischen rhythmus herzustellen sucht. Sie gehören dem spiel von der Kreuzigung an (36). Die hier verwendeten strophen bestehen nicht aus den bisher behandelten vierhebigen versen, aber doch aus einer nahestehenden abart derselben, nämlich dreihebigen, die im übrigen denselben rhythmischen charakter haben. Es sind also gewöhnlich zwei senkungssilben zwischen je zwei hebungen vorhanden, aber auch drei oder nur eine sind möglich; nur völliges fehlen derselben scheint kaum vorzukommen. Offenbar ist dieser vers aus dem vierhebigen durch dieselbe analogiewirkung entstanden, die in der sprachentwicklung eine so grosse rolle gespielt hat. Wie viertaktige verse so häufig mit dreitaktigen wechselten, so stellte man den vierhebigen nun auch dreihebige zur seite. Nebeneinander treten sie uns z. b. in den vorhin berührten stiicken $(1,40)$ entgegen, wo die dreihebigen die zweite strophenhälfte bilden. In der Kreuzigung erscheinen diese neben noch kürzeren und bilden den aufgesang, sowie die letzte zeile des abgesanges (abab bcbc | deeed).

Holthausen will nun in den versen

pat dóulfully to déde pus is dízt $132,325,368$

pus doulffully to déde haue pei dóne 254

statt doulfully ein zweisilbiges wort und zwar derfly einsetzen (eb. s. 449 f.), um das metrum zu bessern. Nach dem dargelegten ist aber dreisilbige senkung in diesem vers historisch berechtigt und in der that zeigt eine durchsicht des stückes, dass sie auch sonst nicht selten vorkommt. Wenn wir sogar von den fällen absehen, wo die flexionsendungen -es, -ed eine der drei senkungssilben bilden (was übrigens kaum nötig wäre, vgl. Angl. XII 452), so bleiben noch folgende unbedingt sichere fälle übrig:

Full spitously to spéde he were spílte 39

Full mádly on pe móne for to mówe 78

pat sódenly has lénte me my sight 302 , 
ZUR TEXTKRITIK DER SPIEIE VON YORK.

ferner $47,188,223,338,383,392,397$. In einem fall :

Who trówes pou, to pi táles toke ténte 81

haben wir sogar vier senkungssilben, wofern nicht etwa doch trowes zu lesen ist. Dazu kommt, dass die verbindung von doulfull mit dede und dye auch sonst vorkommt, und eine stehende formel $\mathrm{zu}$ sein scheint. Man vergleiche z. b.: Was doulfully dight to the dying 429/80; pus with dole was pat dere vn-to dede dight 430/106. Wir werden also auch hier bei den überlieferten lesarten beharren müssen.

GrAz, am 24. Juni 1899.

K. LUICK. 